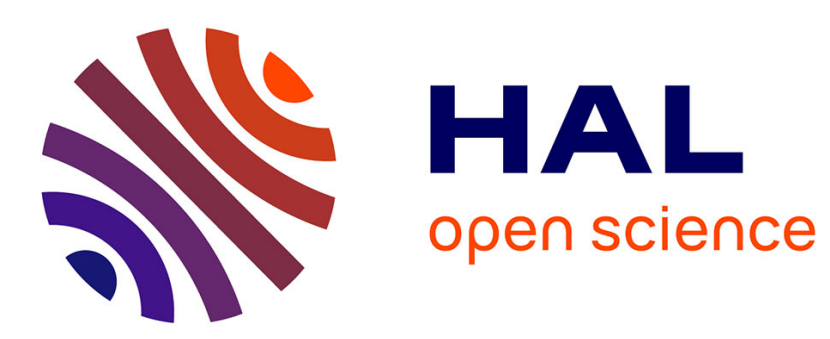

\title{
Effets d'un rayonnement ionisant sur les mécanismes de conduction et de bruit de fond basse fréquence des transistors bipolaires
}

\author{
G. Blasquez, M. Roux-Nogatchewsky
}

\section{- To cite this version:}

G. Blasquez, M. Roux-Nogatchewsky. Effets d'un rayonnement ionisant sur les mécanismes de conduction et de bruit de fond basse fréquence des transistors bipolaires. Revue de Physique Appliquée, 1980, 15 (11), pp.1599-1605. 10.1051/rphysap:0198000150110159900 — jpa-00244887

HAL Id: jpa-00244887

https://hal.science/jpa-00244887

Submitted on 1 Jan 1980

HAL is a multi-disciplinary open access archive for the deposit and dissemination of scientific research documents, whether they are published or not. The documents may come from teaching and research institutions in France or abroad, or from public or private research centers.
L'archive ouverte pluridisciplinaire HAL, est destinée au dépôt et à la diffusion de documents scientifiques de niveau recherche, publiés ou non, émanant des établissements d'enseignement et de recherche français ou étrangers, des laboratoires publics ou privés. 


\title{
Effets d'un rayonnement ionisant sur les mécanismes de conduction et de bruit de fond basse fréquence des transistors bipolaires
}

\author{
G. Blasquez et M. Roux-Nogatchewsky \\ Laboratoire d'Automatique et d'Analyse des Systèmes du Centre National de la Recherche Scientifique, \\ 7, avenue du Colonel-Roche, 31400 Toulouse, France
}

(Reçu le 6 mai 1980, révisé le 4 juillet 1980, accepté le 1er août 1980)

\begin{abstract}
Résumé. - Des transistors bipolaires NPN commandés par effet de champ ont été soumis à des doses comprises entre 10 et $10^{4}$ Gy de rayons $\mathrm{X}$ délivrés par un générateur fonctionnant à $150 \mathrm{kV}$. Des accroissements de grande amplitude de courant de base et du bruit de fond basse fréquence ont été observés quand la surface était appauvrie en porteurs majoritaires ou inversée. Il a été montré que ces accroissements résultaient d'augmentations de la vitesse de recombinaison en surface, de la charge positive située dans l'oxyde et de la densité de pièges donnant le bruit. Des corrélations positives ont été mises en évidence entre les accroissements de ces trois paramètres. Ces résultats ont été attribués à l'ionisation de la silice par des rayons $\mathbf{X}$. Une restauration partielle des caractéristiques des transistors a été obtenue après traitements thermiques. Il a été suggéré qu'il n'existe pas de méthodes spécifiques de durcissement diminuant la sensibilité du bruit au rayonnement.

Abstract. - Gate controlled NPN bipolar transistors were irradiated with doses ranging between 10 and $10^{4} \mathrm{~Gy}$ $X$ rays supplied by a generator functioning at $150 \mathrm{kV}$. An increase of great amplitude of the base current and of the low frequency noise were observed for both depleted and inverted base surface conditions. It has been shown that these increases were due to the enhancement of the total surface recombination velocity, of the positive charge within the oxide and also of the density of noise traps. Positive correlations were observed between these three surface parameters. These results have been attributed to the ionization of silica by $\mathbf{X}$ rays. The transistors partially recovered by means of thermal treatments. It has been suggested that it does not exist specific hardening methods to minimize the noise sensitivity to irradiation.
\end{abstract}

1. Introduction. - Il ressort de travaux récents [1] et [2] effectués sur le bruit de fond de moyenne fréquence des transistors bipolaires soumis à des rayonnements ionisants que le bruit de grenaille et le bruit thermique de la base sont indépendants de la dose reçue et que le bruit de recombinaison produit dans les régions superficielles de la jonction émetteur base croît. Physiquement cet accroissement résulte des effets combinés de deux causes : a) la création des centres de recombinaison et $b$ ) la modification de l'étendue de la charge d'espace de la jonction par des charges électriques positives induites dans la silice.

$A$ priori il est intéressant de se demander si ces rayonnements peuvent influencer le bruit de fond de très basses fréquences dont le spectre suit une loi en $1 / f$ ?

Pour répondre à cette question, il faut se souvenir que dans les transistors bipolaires commandés par effet de champ, l'amplitude de ce bruit varie d'une manière notable quand le potentiel appliqué sur la grille est modifié [3]. Comme l'induction par irradiation d'une charge électrique dans la silice, a le même effet électrostatique que le potentiel de grille, on peut prévoir d'une manière presque sûre une évolution des propriétés du bruit de fond basse fréquence.

L'étude qui suit se propose de montrer l'existence de cette évolution et de la caractériser en précisant les mécanismes physiques sous-jacents. Elle se termine par un examen des problèmes de sensibilité.

2. Etude expérimentale. - 2.1 Conditions DE L'ÉTUDE. - L'étude a été effectuée sur des transistors bipolaires NPN au silicium commandés par effet de champ [3]. Ils se distinguent des transistors bipolaires usuels par la présence d'une métallisation supplémentaire placée sur la couche d'oxyde de silicium recouvrant la jonction émetteur base (voir Fig. 1). L'application d'une tension (dite de grille, et notée dans ce qui suit $V_{\mathrm{G}}$ ) sur cette métallisation permet de faire varier par effet de champ la répartition du potentiel électrostatique à la surface du transistor. On peut simuler ainsi l'influence de la présence de charges électriques dans l'oxyde sur le comportement du transistor et notamment du courant de base et du 


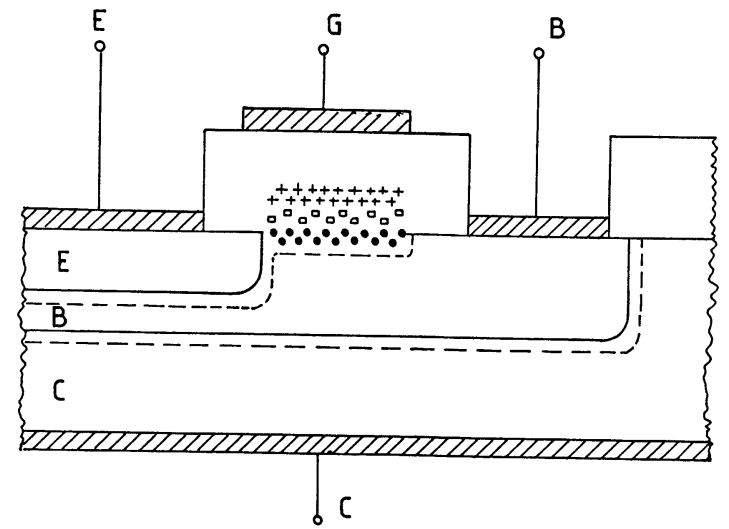

Fig. 1. - La région latérale d'un transistor bipolaire NPN commandable par effet de champ (+ représentent des charges positives, $\square$ des pièges, $\bullet$ des centres de génération recombinaison).

[Lateral region of a gate-controlled NPN bipolar transistor (+ represent positive oxide charges, $\square$ are traps and $\bullet$ are generation recombination centres).]

bruit de fond [4 et 5]. Les principales caractéristiques de ces transistors ont été données dans [2]. Les conditions d'élaboration de l'oxyde ont été les suivantes : température $1050^{\circ} \mathrm{C}$, formation sous atmosphère d'oxygène humide pendant $45 \mathrm{~min}$. et puis sous oxygène sec pendant 5 min., enfin recuit sous argon pendant $90 \mathrm{~min}$. Après gravure des contacts, les transistors sont recuits à $500^{\circ} \mathrm{C}$ sous azote hydrogéné pendant $10 \mathrm{~min}$.

L'épaisseur de l'oxyde sous la grille est de l'ordre de $3000 \AA$. La source de rayons X utilisée pour cette étude était un générateur du type AequiVolt 300 fabriqué par C.G.R. Ses conditions nominales de fonctionnement étaient : tension d'alimentation $150 \mathrm{kV}$, courant continu $10 \mathrm{~mA}$, dose délivrée par minute en régime continu $10 \mathrm{~Gy}\left({ }^{1}\right)$.

Les paramètres mesurés étaient $: a$ ) les caractéristiques statiques et $b$ ) le générateur de bruit en courant $\left\langle\left|i_{\mathrm{F}}\right|^{2}\right\rangle$ placé à l'entrée du transistor en fonction de la tension $V_{\mathrm{G}}$ appliquée sur la grille pour un point de polarisation fixé (tension émetteur base $V_{\mathbf{B E}}$, tension collecteur base $V_{\mathrm{CE}}$ et température $T$ constantes). Les mesures de bruit de fond ont été effectuées avec les équipements et suivant la méthode décrits dans [4], dans une gamme de fréquence s'étendant de $10 \mathrm{~Hz}$ à $5 \mathrm{kHz}$.

2.2 RéSultats. - Après irradiation, on a observé d'une manière générale que pour un point de polarisation donné, le courant collecteur est indépendant de la dose reçue tandis que le courant de base croît. Un exemple typique de variations du courant de base en fonction de la tension de grille est donné sur la figure 2. Pour ce transistor les mesures ont été effectuées à $V_{\mathrm{BE}}=0,56 \mathrm{~V}, V_{\mathrm{CE}}=2 \mathrm{~V}, T=294 \mathrm{~K}$. La

( $\left.{ }^{1}\right) 1 \mathrm{~Gy}=100$ rads. Les doses mentionnées dans [2] doivent être divisées par $10^{4}$. Cette erreur est due à l'utilisation de la formule de conversion donnée dans Physica 93A (1978) 49.

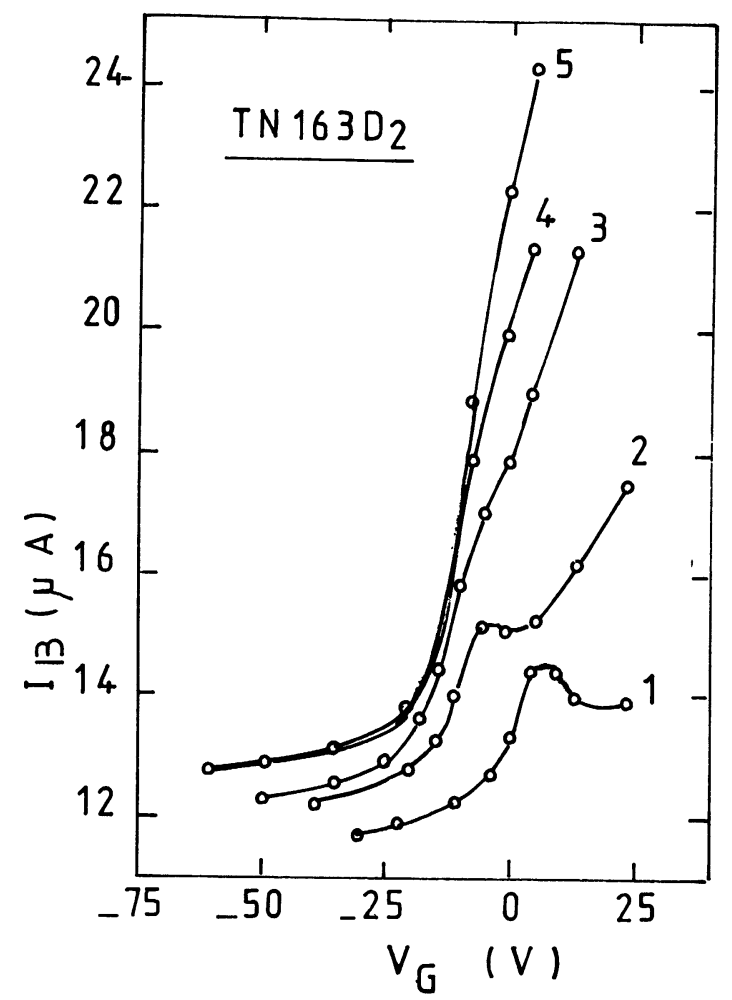

Fig. 2. - Le courant de base en fonction de la tension de grille avant et après irradiation : 1) avant, 2) après $\left.3 \times 10^{2} \mathrm{~Gy}, 3\right) 10^{3} \mathrm{~Gy}$, 4) $\left.3 \times 10^{3} \mathrm{~Gy}, 5\right) 10^{4} \mathrm{~Gy}$. Conditions de polarisation : $I_{\mathrm{C}}=1 \mathrm{~mA}$, $V_{\mathrm{BE}}=0,56 \mathrm{~V}$ et $T=294 \mathrm{~K}$.

[The base current $v s$. gate voltage before and after irradiation : 1) before, 2) after $\left.\left.3 \times 10^{2} \mathrm{~Gy}, 3\right) 10^{3} \mathrm{~Gy}, 4\right) 3 \times 10^{3} \mathrm{~Gy}$, 5) $10^{4} \mathrm{~Gy}$. Biasing conditions : $I_{\mathrm{C}}=1 \mathrm{~mA}, V_{\mathrm{BE}}=0.56 \mathrm{~V}, T=294 \mathrm{~K}$.]

caractéristique avant irradiation porte le numéro un. Les courbes 2 à 5 ont été obtenues après des doses respectivement égales à : $3 \times 10^{2} \mathrm{~Gy}, 10^{3} \mathrm{~Gy}, 3 \times 10^{3} \mathrm{~Gy}$ et $10^{4} \mathrm{~Gy}$.

Pour les doses faibles, le maximum du courant de base, noté dans ce qui suit $I_{\mathrm{B}, \mathrm{M}}$, croît tandis que la tension de grille correspondante, notée $V_{\mathrm{G} \text { peak }}$, se déplace vers les valeurs négatives.

Pour les doses élevées, le maximum s'estompe et souvent disparaît. En valeur relative, les accroissements du courant sont faibles pour les tensions de grille négatives, c'est-à-dire quand la surface de la base est en régime d'accumulation [5]. Ils sont élevés pour des tensions de grille positives, c'est-à-dire quand la surface est appauvrie en porteurs majoritaires ou quand il existe une couche d'inversion. Des observations similaires ont été reportées dans [6]. Le bruit de fond de très basse fréquence est lui aussi modifié par les rayons $X$. Son spectre qui suivait une loi en $1 / f$ avant irradiation évolue vers une loi en $1 / f^{\alpha}$ avec $0,8<\alpha<1,2$. Dans de nombreux cas, ces variations ne sont pas significatives à cause de la précision limitée des mesures de bruit (de l'ordre de $15 \%$ ). Les résultats des mesures effectuées à fréquence constante sont plus intéressants. Un exemple typique est donné sur la figure 3. Le repérage des courbes est identique à celui utilisé sur la figure 2. D'une manière générale, 


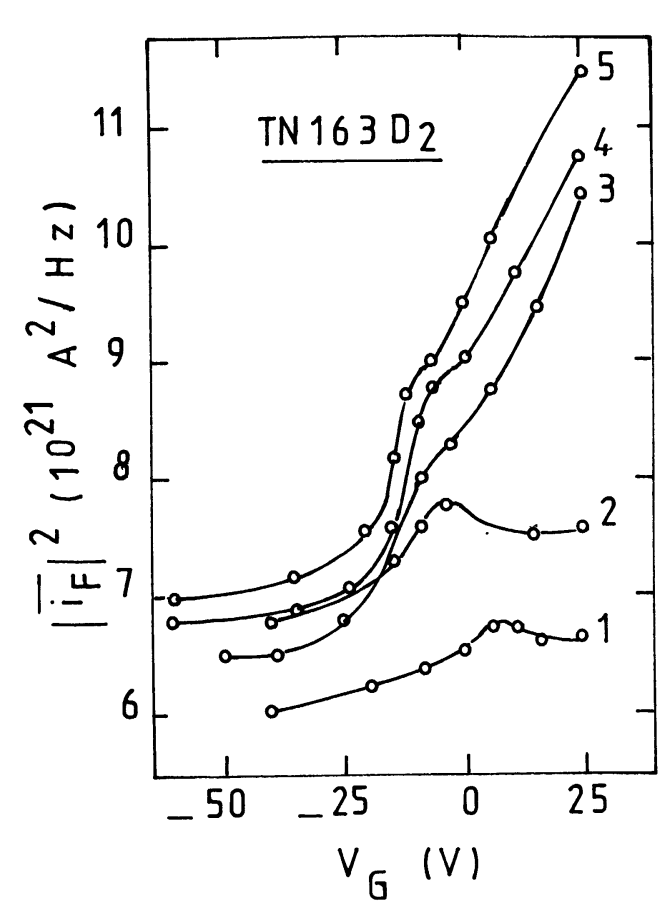

Fig. 3. - Le bruit en $1 / f$ en fonction de la tension de grille avant et après irradiation. Les doses de rayons $\mathrm{X}$ sont indiquées sur la figure 2. Conditions de mesure $I_{\mathrm{C}}=1 \mathrm{~mA}, T=294 \mathrm{~K}, f=80 \mathrm{~Hz}$.

[The $1 / f$ noise $v s$. gate voltage before and after irradiation. $\mathrm{X}$ rays doses are given in figure 2 . Measurement conditions : $I_{\mathrm{C}}=1 \mathrm{~mA}$, $T=294 \mathrm{~K}, f=80 \mathrm{~Hz}$.]

les variations du bruit ressemblent à celles du courant de base : le maximum croît quand la dose augmente et la tension de grille associée à ce maximum se déplace vers les valeurs négatives. Les accroissements du bruit sont importants quand la surface de la base est en régime d'appauvrissement ou d'inversion.

3. Analyse des résultats expérimentaux. - Il a été montré dans [2] que les rayons $X$ ne modifient pas les propriétés volumiques des transistors. Leurs effets principaux se produisent plutôt au niveau des régions superficielles. Par intégration de la loi de Gauss à la surface de la base, en dehors de la jonction métallurgique émetteur base, il peut être établi que la tension $V_{\mathrm{G} \text { peak }}$ associée au maximum du courant de base est donnée par la relation [4] :

$$
\begin{aligned}
V_{\mathrm{G} \text { peak }}=-\frac{Q_{\mathrm{SS}}}{C_{0 \mathrm{x}}}+\varphi_{\mathrm{S} \mathrm{peak}} & +\varphi_{\mathrm{MS}}+ \\
+ & \frac{1}{C_{0 \mathrm{x}}}\left(2 q \varepsilon_{0} K_{\mathrm{S}} N_{\mathrm{A}} \varphi_{\mathrm{S} \text { peak }}\right)^{1 / 2}
\end{aligned}
$$

où

$$
\varphi_{\mathrm{S} \text { peak }}=\frac{k T}{q} \ln \left(\frac{N_{\mathrm{A}}}{n_{\mathrm{i}}}\right)-\frac{V_{\mathrm{EB}}}{2},
$$

$k, T, q, n_{\mathrm{i}}$ ont leurs significations usuelles,

$Q_{\text {ss }}$ est la densité par unité de surface des charges localisées dans la silice,

$C_{0 \mathrm{x}}$ est la capacité par unité de surface de la silice,
$\varphi_{\mathrm{S} \text { peak }}$ est la valeur du potentiel de surface associée à $V_{\text {G peak }}$,

$N_{\mathrm{A}}$ est la concentration en impuretés de dopage à la surface de la base,

$\varphi_{\text {MS }}$ est la différence des travaux de sortie entre le métal de grille et le silicium,

$\varepsilon_{0}$ est la permittivité du vide,

$K_{\mathrm{S}}$ est la constante diélectrique du silicium.

Dans l'établissement de la relation (1) il a été supposé en première approximation que les charges dans la silice sont localisées près de l'interface $\mathrm{Si}_{-} \mathrm{SiO}_{2}$. Elles ont plusieurs origines physiques possibles : les contaminations ioniques, des complexes à base de silicium, des pièges et des centres de génération recombinaison (voir Fig. 1).

Il est communément admis [7 à 10] que les rayons $\mathrm{X}$ n'ont aucun effet sur les paramètres comme $K_{\mathrm{S}}, N_{\mathrm{A}}$, $\varphi_{\mathrm{MS}}, C_{0 \mathrm{x}}$. Il s'ensuit que la dérive de $V_{\mathrm{G} \text { peak }}$ vers les valeurs négatives de $V_{\mathrm{G}}$ est le résultat de l'induction d'une charge positive $Q_{\text {ss }}$ dans la silice. Deux exemples de variations de cette charge en fonction de la dose reçue par les transistors sont montrés sur la figure 4. Pour des doses inférieures à $10^{3}$ Gy la croissance de $Q_{\text {ss }}$ est rapide. Au-delà un phénomène de saturation apparaît. Dans tous les cas, la saturation s'est produite à des densités de l'ordre de quelque $10^{12}$ charges par $\mathrm{cm}^{2}$.

La croissance de $I_{B, M}$ et les déformations des courbes $I_{\mathrm{B}}\left(V_{\mathrm{G}}\right)$ montrent que les rayons $\mathrm{X}$ ont provoqué en outre une augmentation des courants de recombinaison prenant naissance à la surface de la jonction émetteur base. Pour préciser l'origine physique de cette augmentation, on peut caractériser les variations du courant par le paramètre $s_{0}^{*}$ défini par :

$$
s_{0}^{*}=\frac{2\left(I_{\mathrm{B}, \mathrm{M}}-I_{\mathrm{B}, \mathrm{MJ}}\right)}{q n_{\mathrm{i}} A_{\mathrm{S}} \exp \left(\frac{q V_{\mathrm{EB}}}{2 k T}\right)}
$$

où $I_{\mathrm{B}, \mathrm{MJ}}$ est la valeur du courant de base quand le potentiel de surface est nul (en pratique c'est la valeur minimale de la courbe $\left.I_{\mathrm{B}}\left(V_{\mathrm{G}}\right)\right), A_{\mathrm{S}}$ est l'aire de la zone

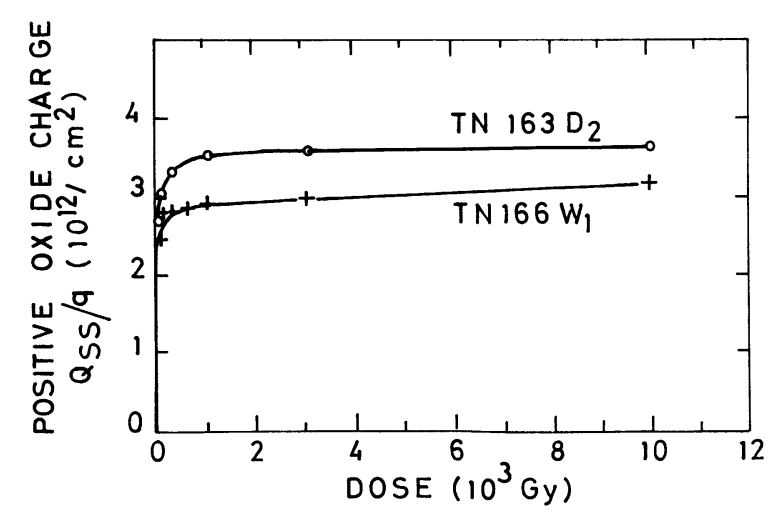

Fig. 4. - La charge dans l'oxyde en fonction de la dose d'irradiation pour deux transistors.

[The oxide charge $v s$. irradiation dose for two transistors.] 
de charge d'espace superficielle induite à la surface par $V_{\mathrm{G}}$ et $Q_{\mathrm{SS}}$.

La signification physique de $s_{0}^{*}$ est la suivante : au voisinage de $I_{\mathrm{B}, \mathrm{M}}$ le courant de diffusion en surface est négligeable par rapport aux courants de recombinaison. Le paramètre $s_{0}^{*}$ est alors lié à la notion courante de vitesse de recombinaison en surface $s_{0}$ par :

$$
s_{0}^{*}=s_{0}+\frac{y_{\mathrm{d}}}{\tau_{0, \mathrm{FISC}}}
$$

où $y_{\mathrm{d}}$ est l'épaisseur de la charge d'espace superficielle induite par $V_{\mathrm{G}}$ et $Q_{\mathrm{ss}}$ dans la base,

$\tau_{0, \text { FISC }}$ est la durée de vie des électrons dans cette zone.

Etant donné que $s_{0}$ caractérise les centres de recombinaison situés à l'interface $\mathrm{Si}_{-} \mathrm{SiO}_{2}$ et que $\tau_{0, \text { FISC }}$ caractérise ceux situées à l'intérieur de la charge d'espace, il est clair que $s_{0}^{*}$ est une caractéristique de l'ensemble des centres de recombinaison en surface. Par extension nous désignerons $s_{0}^{*}$ par : vitesse de recombinaison totale à la surface.

Les variations de $s_{0}^{*}$ pour deux transistors en fonction de la dose sont montrées sur la figure 5. L'allure des courbes ressemble à celle de la figure 4 mais le phénomène de saturation est moins apparent. Si l'on

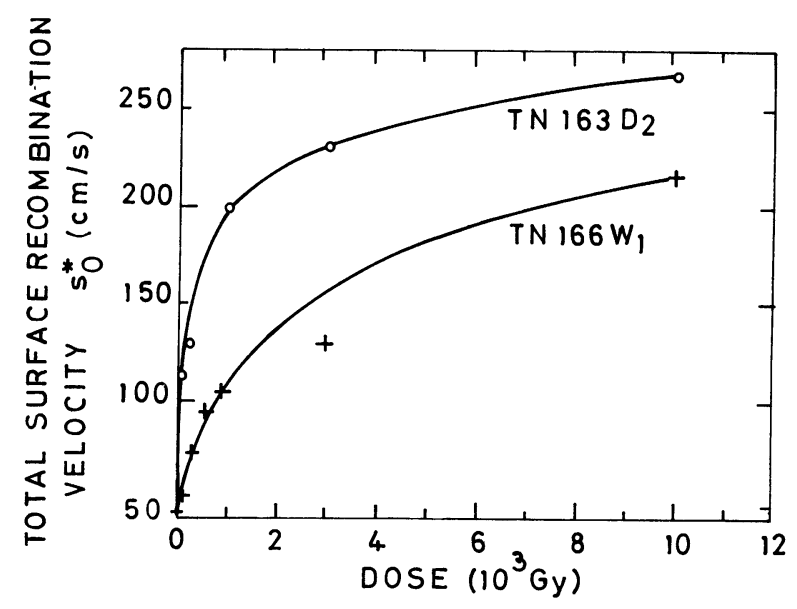

Fig. 5. - Variation de la vitesse de recombinaison totale en surface en fonction de la dose d'irradiation.

[Variations of the total surface recombination velocity as a function of irradiation dose.]

se souvient que la présence d'un maximum dans les courbes $I_{\mathrm{B}}\left(V_{\mathrm{G}}\right)$ indique que le courant de recombinaison associé aux centres d'interface est supérieur au courant donné par les centres de la zone de charge d'espace, il découle des figures 5 et 2 que la croissance de $s_{0}^{*}$ aux faibles doses est due principalement à celle de $s_{0}$. Par contre l'effacement progressif du maximum de $I_{\mathrm{B}}$ et le phénomène de saturation de $Q_{\mathrm{ss}}$ incitent à penser que pour les fortes doses d'irradiation, la croissance lente de $s_{0}^{*}$ provient de $\tau_{0 \text {,Fisc }}$. Autrement dit, $s_{0}$ présenterait comme $Q_{\mathrm{ss}}$ une saturation.

Pour analyser les résultats en bruit de fond, nous postulons que le mécanisme physique sous-jacent est une modulation des composantes superficielles du courant de base par des pièges situés dans la silice près de l'interface $\mathrm{Si}_{-} \mathrm{SiO}_{2}$ [11]. Le calcul de ce bruit ne peut être effectué que sur calculatrice arithmétique quand tous les paramètres caractéristiques de la surface et des pièges sont connus [4]. En général ce n'est pas le cas, et on est réduit à utiliser un modèle grossier [11] qui indique que le bruit prenant naissance dans la charge d'espace induite à la surface, quand celle-ci n'est pas trop appauvrie de porteurs majoritaires, est égal à :

$$
\begin{aligned}
\left\langle\left|i_{\mathrm{F}}\right|^{2}\right\rangle \simeq 2 q^{2} \frac{1}{\varepsilon_{0} N_{\mathrm{A}} K_{\mathrm{S}}} \frac{q \varphi_{\mathrm{S}}}{k T} & y_{0} N_{\mathrm{t}} \frac{1}{A_{\mathrm{S}}} \times \\
& \times\left(I_{\mathrm{B}}-I_{\mathrm{B}, \mathrm{MJ}}\right)^{2} \frac{\Delta f}{f}
\end{aligned}
$$

où $N_{\mathrm{t}}$ est la densité par unité d'énergie et de volume des pièges,

$\varphi_{\mathrm{S}}$ est le potentiel de surface,

$f$ la fréquence,

$y_{0}^{-1}$ est un facteur de l'ordre de $1,5 \times 10^{8} \mathrm{~cm}^{-1}$ (voir [11]).

La dépendance du bruit au courant de base explique les similitudes observées entre les figures 2 et 3 et il est clair que la première cause de l'augmentation du bruit après irradiation est due aux variations de $I_{\mathrm{B}}$. Il est alors intéressant de rechercher si les rayons $X$ ont influencé $N_{\mathrm{t}}$. Etant donné qu'il est admis que $\varepsilon_{0}, N_{\mathrm{A}}$, $K_{\mathrm{S}}, y_{0}, A_{\mathrm{S}}$ sont des paramètres indépendants du rayonnement, l'expression (4) montre que l'on peut caractériser les variations de $N_{\mathrm{t}}$ par la quantité $\left\langle\left|i_{\mathrm{F}}\right|^{2}\right\rangle / q \varphi_{\mathrm{S}} / k T$ estimée à $f, \Delta f$ et $\left(I_{\mathbf{B}}-I_{\mathrm{B}, \mathrm{MJ}}\right)$ constants. En pratique $\left\langle\left|i_{\mathrm{F}}\right|^{2}\right\rangle$ à $f, \Delta f$ et $\left(I_{\mathrm{B}}-I_{\mathrm{B}, \mathrm{MJ}}\right)$ constants est déduit des figures 2 et 3 , tandis que $\varphi_{\mathrm{S}}$ est évalué par résolution de l'équation de neutralité (équation (1) dans laquelle on remplace $V_{\mathrm{G} \text { peak }}$ par $V_{\mathrm{G}}, \varphi_{\mathrm{S} \text { peak }}$ par $\varphi_{\mathrm{S}}$ et $Q_{\mathrm{SS}}$ est remplacé par sa valeur obtenue quand $V_{\mathrm{G}}=V_{\mathrm{G} \text { peak }}$ ). Les résultats obtenus. malgré une dispersion assez importante montrent que $N_{\mathrm{t}}$ est également une fonction croissante de la dose.

Les figures 6 et 7 montrent l'évolution du paramètre indicatif des variations de $N_{\mathrm{t}}$ en fonction d'une part de $Q_{\text {ss }}$ et d'autre part de $s_{0}^{*}$. Une corrélation positive apparaît clairement dans les deux cas. Pour le transistor $\mathrm{TN}_{163 \mathrm{D}_{2}}$ une relation linéaire existe entre $N_{1}$ et $s_{0}^{*}$. Ceci signifie que la capture des porteurs du semiconducteur par les pièges s'effectue via les centres de recombinaison comme l'avait proposé $\mathrm{Fu}$ et $\mathrm{Sah}$ [12]. La dispersion observée sur les figures provient de plusieurs causes : a) les limitations des modèles pour décrire d'une manière précise les mécanismes de conduction et de bruit à la surface, $b$ ) les incertitudes sur les mesures de bruit, $c$ ) les approximations effectuées dans le calcul de $\varphi_{\mathrm{S}}, d$ ) l'existence de faibles dérives temporelles des caractéristiques du transistor après irradiation. 

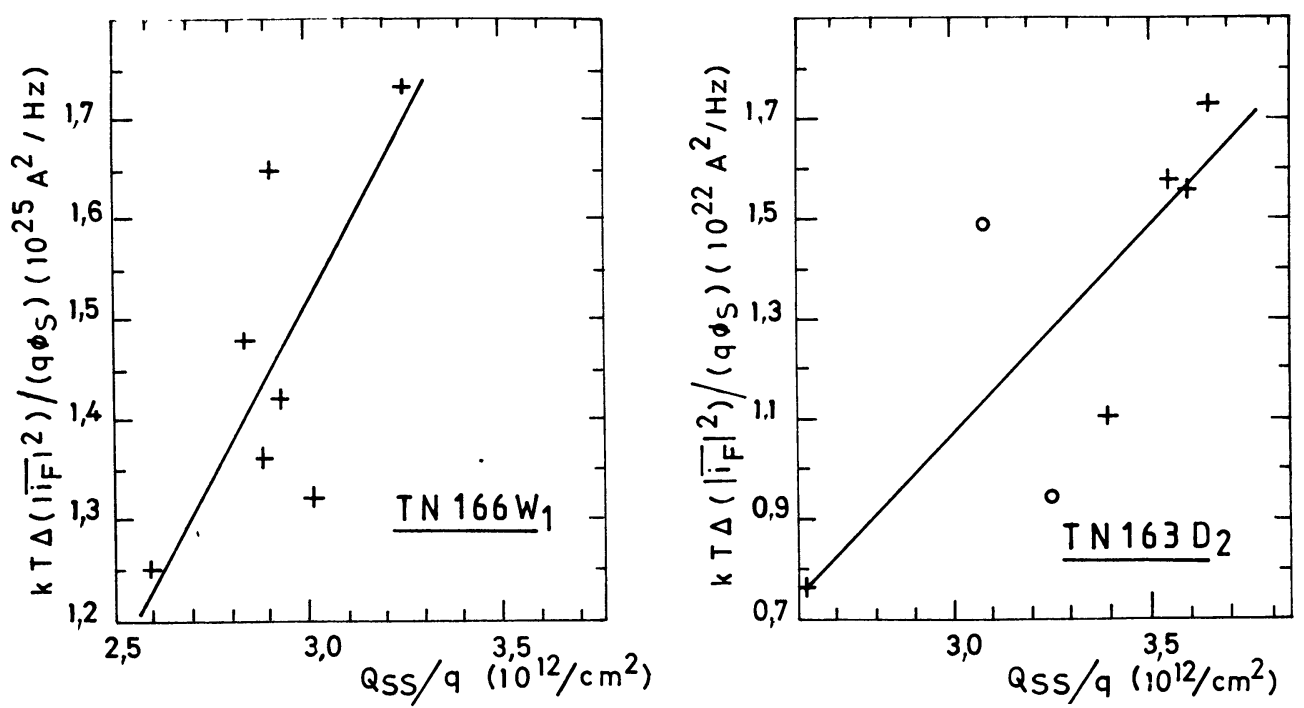

Fig. 6. - Correlation entre les accroissements de bruit en $1 / f$ et la charge dans l'oxyde. Conditions expérimentales : transistor TN166W1, $I_{\mathrm{B}}-I_{\mathrm{B}, \mathrm{MJ}}=0,6 \mu \mathrm{A}, f=80 \mathrm{~Hz}, I_{\mathrm{C}}=100 \mu \mathrm{A}, T=294 \mathrm{~K}$, transistor TN163D2, $I_{\mathrm{B}}-I_{\mathrm{B}, \mathrm{MJ}}=2,72 \mu \mathrm{A}, f=80 \mathrm{~Hz}, I_{\mathrm{C}}=1 \mathrm{~mA}, T=294 \mathrm{~K}$

[Correlation between the $1 / f$ noise increases and the charge within the oxide layer. Experimental conditions : transistor TN166W1. $I_{\mathrm{B}}-I_{\mathrm{B}, \mathrm{MJ}}=0.6 \mu \mathrm{A}, f=80 \mathrm{~Hz}, I_{\mathrm{C}}=100 \mu \mathrm{A}, T=294 \mathrm{~K}$. Transistor TN163D2, $I_{\mathrm{B}}-I_{\mathrm{B}, \mathrm{MJ}}=2.72 \mu \mathrm{A}, f=80 \mathrm{~Hz}, I_{\mathrm{C}}=1 \mathrm{~mA}, T=294 \mathrm{~K}$.]
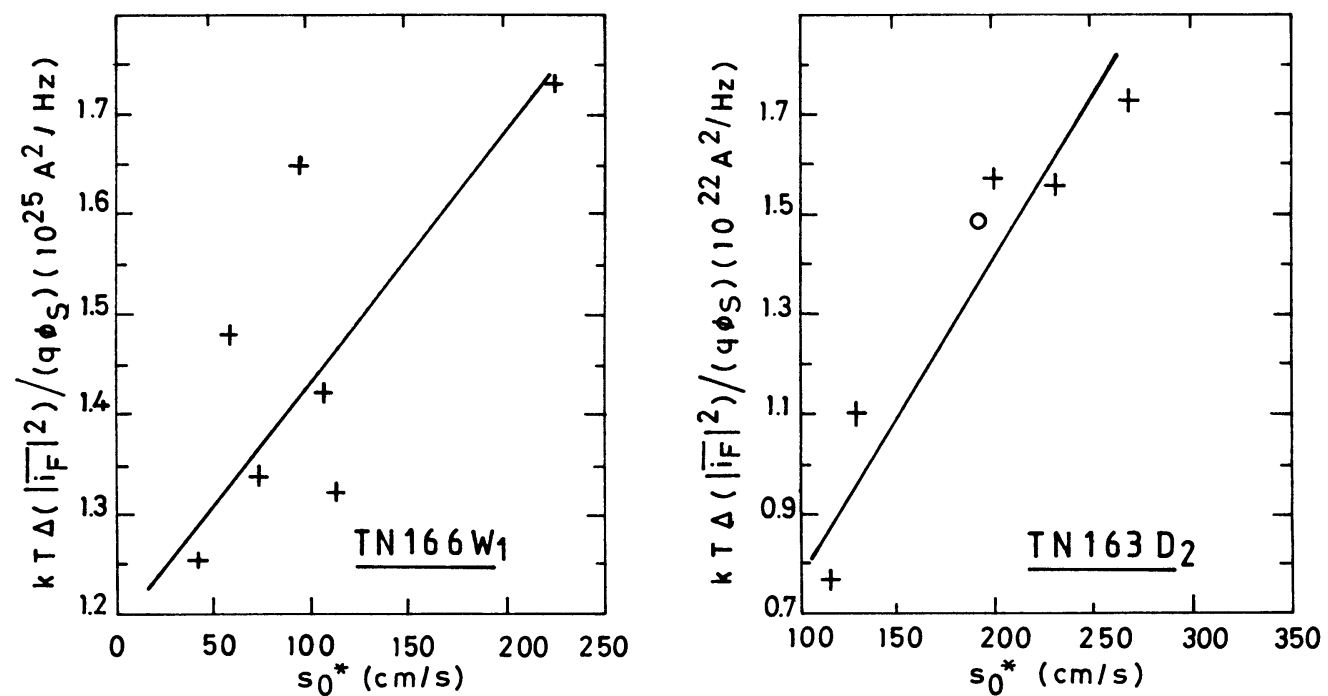

Fig. 7. - Corrélation entre les accroissements du bruit en $1 / f$ et la vitesse totale de recombinaison en surface. Les conditions expérimentales sont indiquées sur la figure 6 .

[Correlation between the $1 / f$ noise increases and the total surface recombination velocity. Experimental conditions are given in figure 6.]

4. Interprétation des résultats. - L'effet principal des rayons $\mathrm{X}$ est d'ioniser la couche de silice [7 à 10]. Une partie des électrons ainsi produits se recombinent aux interfaces grille-silice et silice-silicium. Pour simplifier nous supposons qu'après irradiation, le mécanisme prépondérant d'échange de charges entre le silicium et la silice s'effectue par effet tunnel sur une distance de l'ordre de $30 \AA$. Dans ces conditions, les ions induits par le rayonnement peuvent se comporter de trois manières différentes. Ceux situés à plus de $30 \AA$ de l'interface $\mathrm{Si}_{-} \mathrm{SiO}_{2}$ ne peuvent pas communiquer avec le semiconducteur. Ils apparaissent comme des charges positives (croix de la Fig. 1). Ils sont responsables de l'augmentation de $Q_{\mathrm{ss}}$. Les ions créés à l'interface $\mathrm{Si}_{-} \mathrm{SiO}_{2}$ peuvent capturer ou émettre des électrons et des trous. Dans ce cas, ils se comportent comme des centres de recombinaison. Cette catégorie (ronds de la Fig. 1) provoque la croissance de $s_{0}^{*}$. Enfin, la dernière catégorie (carrés de la Fig. 1) est constituée par les ions induits dans la zone intermédiaire. Ils se distinguent des précédents par des constantes de temps plus élevées. Ils constituent principalement des sources de bruit. Ce modèle simple rend compte des corrélations observées entre $N_{\mathrm{t}}, Q_{\mathrm{ss}}$ et $s_{0}^{*}$.

Il implique aussi que tout traitement réduisant la densité des ions induits par le rayonnement doit pro- 


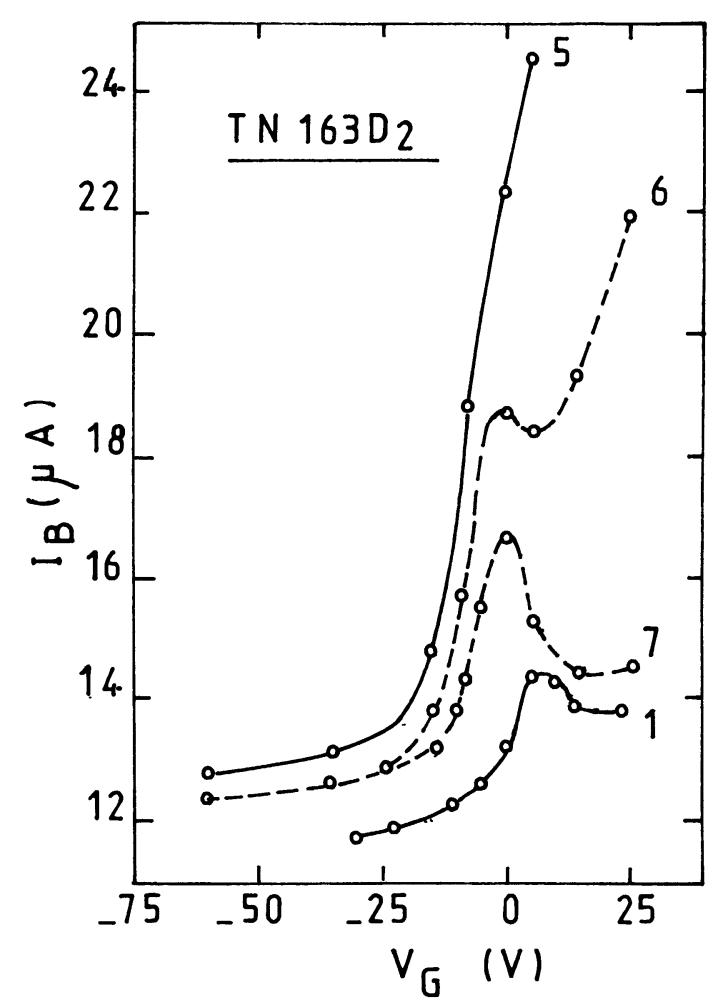

Fig. 8. - Effet des traitements thermiques après irradiation sur le courant de base à $I_{\mathrm{C}}=1 \mathrm{~mA}, T=294 \mathrm{~K}$. Courbe 1 : avan! irradiation, 5) après une dose d'irradiation de $\left.10^{4} \mathrm{~Gy}, 6\right)$ aprì recuit de $100 \mathrm{~min}$. à $\left.150^{\circ} \mathrm{C}, 7\right)$ après $60 \mathrm{~min}$. à $300^{\circ} \mathrm{C}$.

[The effect of thermal treatments after irradiation on base current at $I_{\mathrm{C}}=1 \mathrm{~mA}, T=294 \mathrm{~K}$. Curve 1 : before irradiation, 5) after an irradiation dose of $\left.10^{4} \mathrm{~Gy}, 6\right)$ after heating the transistor for $100 \mathrm{~min}$. at $\left.150^{\circ} \mathrm{C}, 7\right)$ after $60 \mathrm{~min}$. at $300^{\circ} \mathrm{C}$.]

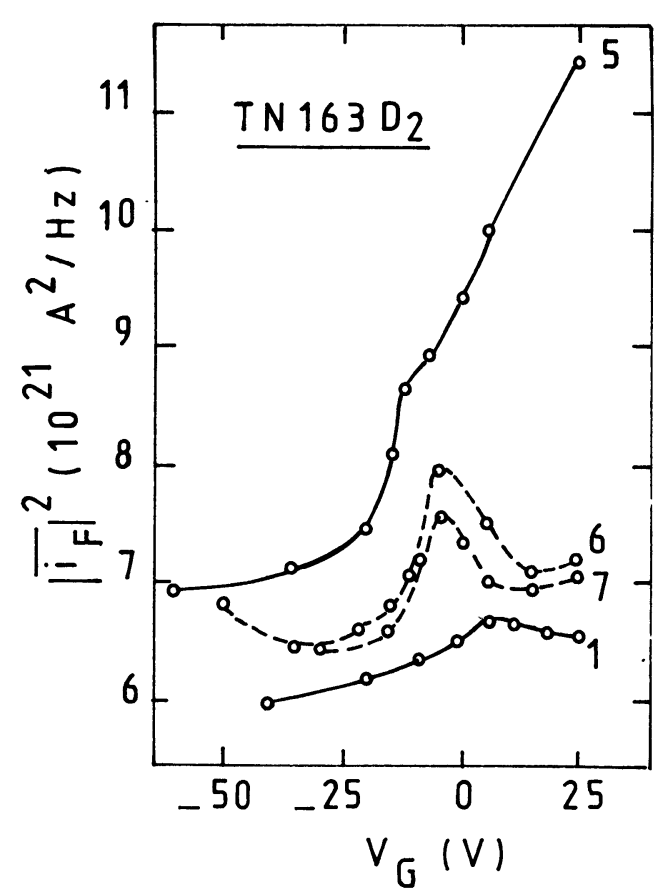

Fig. 9. - Effet des traitements thermiques après irradiation sur le bruit en $1 / f$. Les conditions expérimentales sont données en figure 8.

[The effect of thermal treatments after irradiation on $1 / f$ noise. Experimental conditions are given in figure 8.] voquer une diminution du courant de base et du bruit. Pour vérifier cette conséquence du modèle, les transistors ont été recuits à $150^{\circ} \mathrm{C}$ pendant $100 \mathrm{~min}$. et à $300^{\circ} \mathrm{C}$ pendant $60 \mathrm{~min}$. avec les électrodes en court circuit. Un exemple de résultats obtenus est donné sur les figures 8 et 9 . Les courbes portent les numéros 1 à 7. Ils indiquent respectivement : 1) les caractéristiques avant irradiation, 5) l'état après irradiation avec $\left.10^{4} \mathrm{~Gy}, 6\right)$ l'état après irradiation et le premier recuit, 7) l'état après irradiation et les deux recuits. Il apparaît que les traitements thermiques réduisent les dégradations provoquées par les rayons $\mathrm{X}$. La dérive de $V_{\mathrm{G} \text { peak }}$ vers les tensions positives de $V_{\mathrm{G}}$ indique que $Q_{\text {ss }}$ décroît. La restauration de pic du courant base et la diminution de son amplitude mettent en évidence une réduction de $s_{0}^{*}$ et de $\tau_{0 \text {,FISc }}$. L'analyse des courbes de bruit montrent une décroissance de $N_{\mathrm{t}}$ conformément aux déductions précédentes.

5. Conséquences pratiques. - Dans [2] il avait été observé que le bruit mesuré à quelques dizaines de $\mathrm{kHz}$ et le courant de base induits par les rayons $\mathrm{X}$ sont proportionnels. Il a été mentionné dans ce qui précède que le bruit de très basse fréquence est influencé par le rayonnement non seulement par l'intermédiaire de $I_{\mathrm{B}}$, mais aussi à cause des variations de $N_{\mathrm{t}}$. D'une manière générale, la sensibilité du bruit de basse fréquence est donc plus importante que celle du bruit de moyenne fréquence. En ce qui concerne le durcissement, il avait été conclu dans [2] qu'il n'existe pas de méthodes spécifiques pour réduire la sensibilité du bruit de recombinaison au rayonnement. Les corrélations observées entre les évolutions de $N_{\mathrm{t}}$ et de $s_{0}^{*}$ suggèrent que la même conclusion s'applique au bruit en $1 / f$ des transistors dont la couche d'oxyde superficiel est élaborée suivant les conditions mentionnées dans la section 2.1. Il serait alors intéressant de poursuivre l'étude sur des transistors fabriqués différemment pour préciser le domaine de validité de la suggestion précédente.

6. Conclusion. - Le courant de base et le bruit de fond en $1 / f$, des transistors bipolaires contrôlés par effet de champ soumis à un rayonnement $\mathrm{X}$ délivré d'une manière continue par un générateur fonctionnant à $150 \mathrm{kV}, 10 \mathrm{~mA}$, augmentent pour des doses supérieures à $10 \mathrm{~Gy}$. Ces augmentations sont importantes quand la surface de la base est appauvrie de porteurs majoritaires ou inversée. La dérive du courant de base est due à l'induction d'une charge positive fixe dans l'oxyde et à l'augmentation de la vitesse de recombinaison en surface. Celle du bruit de fond provient non seulement de l'évolution du courant de base, mais aussi de l'induction de sources microscopiques de bruit en $1 / f$. L'existence de corrélations positives entre les accroissements respectifs de la charge, de la vitesse de recombinaison et de la densité des sources de bruit suggèrent qu'ils résultent d'un seul mécanisme physique : l'ionisation de la couche 
d'oxyde superficiel par les rayons X. Les ions créés, seraient tous de même nature physique, mais les effets sur les paramètres du transistor seraient différents suivant leurs positions énergétiques et spatiales. Les dérives du courant de base et du bruit induits par le rayonnement sont partiellement réduites par recuits thermiques.

Au plan des applications, il faut souligner que la sensibilité au rayonnement du bruit de très basse fréquence est plus élevée que celle du bruit de moyenne fréquence. Néanmoins les suggestions qui découlent des résultats présentés dans ce qui précède sont les mêmes que celles qui avaient été données dans [2]. Ceci est une conséquence des corrélations observées.

Enfin il apparaît que l'emploi des rayons $\mathrm{X}$ et des recuits constituent une technique fructueuse pour les études fondamentales sur le bruit en $1 / f$.

Remerciements. - Les auteurs remercient G. Pierrel, J. Chevallier, F. Rossel et P. Fadel d'avoir fabriqué les transistors commandables par effet de champ.

Ils remercient également les rapporteurs d'avoir suggéré des améliorations au manuscrit.

\section{Bibliographie}

[1] KeRnS and Chen, I.E.E.E. Trans. Nucl. Sci. 18 (1971) 37.

[2] Blasquez, G. et Roux-Nogatchewsky, M., Revue Phys. Appl. 14 (1979) 821.

[3] SAH, C. T., Proc. I.R.E. 49 (1961) 1623.

[4] Blasquez, G., Thèse de Doctorat ès Sciences Physiques, no. 532, Université Paul-Sabatier, Toulouse (1973).

[5] Grove, A. S., Physics and Technology of Semiconductor Devices (Wiley, New York) 1967.

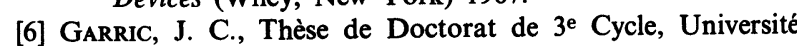
Paul-Sabatier, Toulouse (1974).
[7] Snow, E. H., Grove, A. S. and Fitzgerald, D. J., Proc. I.E.E.E. 55 (1967) 1168.

[8] KoII, E., I.E.E.E. Trans. Electron Devices 13 (1966) 12

[9] Hughes, H. L., I.E.E.E. Trans. Nucl. Sci. 16 (1969) 195.

[10] Reves, A. G. and Zaininger, K. H., RCA Rev. 3 (1968) 22.

[11] Blasquez, G., Phys. Status Solidi (a) 34 (1976) 207.

[12] FU, H. S. and SAH, C. T., I.E.E.E. Trans. Electron Devices 19 (1972) 273. 